\title{
Implicaciones de la pandemia por COVID-19 en la salud docente. Revisión sistemática*
}

\author{
Implications of the COVID-19 pandemic on the health of \\ teachers. Systematic review
}

Implicações da pandemia de COVID-19 na saúde docente. Revisão sistemática

\section{Evangelina Cervantes Holguín**}

\section{RESUMEN}

En el siglo XXI, la docencia representa una profesión que se configura entre exigencias diversas. En marzo del 2020, el profesorado se enfrentó a un inédito giro provocado por COVID-19 que impulsó el teletrabajo y con ello nuevas problemáticas alrededor de la salud docente. Así, el ejercicio se realizó en torno a tres preguntas: ¿qué dice la investigación respecto de la salud/enfermedad del profesorado en tiempos de la COVID-19?, ¿cómo viven los docentes la profesión en el contexto de pandemia?, y ¿qué experiencias pueden orientar el diseño de políticas sociales para el bienestar docente en momentos de incertidumbre? El trabajo se elaboró desde la sociología de los cuerpos/emociones como perspectiva analítica y la revisión bibliográfica sistematizada como perspectiva metodológica. La revisión de los 26 artículos implicó la realización de cuatro actividades: búsqueda, evaluación, análisis y síntesis. Del estudio se concluye que la salud/ enfermedad docente constituye un tópico recurrente en la investigación internacional. El teletrabajo, como política educativa,
Palabras clave: docente, pandemia, política de la salud, salud, salud mental.

* La presente experiencia de investigación procede del proyecto en curso referente a la salud docente del profesorado de educación básica en el norte de México. Las reflexiones aquí expuestas son responsabilidad única y exclusiva de la autora del presente trabajo.

** Mexicana. Doctora en Educación por el Centro Universitario de Tijuana, México. Académica de la Universidad Autónoma de Ciudad Juárez. Chihuahua, México. evangelina.cervantes@uacj.mx 
trastocó la vida personal, profesional y familiar del profesorado con efectos en su cuerpo/emociones. La evidencia da cuenta de una diversidad de intervenciones que pueden ser consideradas en el diseño de políticas docentes en favor de su salud integral en tiempos de pandemia.

\begin{abstract}
In the 21st century, teaching has come to represent a profession that is shaped by a variety of demands. In March 2020, teachers were faced with an unprecedented situation caused by COVID-19, which promoted teleworking and brought with it new health-related problems for teachers. Thus, the work was based on three questions: what does research say about teachers' health/illness in times of COVID-19; how do teachers cope during the pandemic; and what experiences can guide the design of social policies for teachers' wellbeing in times of uncertainty? The work was elaborated from the sociology of bodies/emotions as an analytical perspective and the systematized bibliographic review as a methodological perspective. The review of the 26 articles involved four activities: search, evaluation, analysis and synthesis. The study concluded that teachers' health/illness becomes a recurrent topic in international research. Telework, as an educational policy, disrupted the personal, professional and family lives of teachers and affected their bodies/emotions. The evidence shows a diversity of actions that can be taken when designing teaching policies that support their overall health in times of pandemic.
\end{abstract}

\section{RESUMO}

A docência no século XXI representa uma profissão moldada por diversas exigências. Em março de 2020, a classe docente foi confrontada por uma mudança sem precedentes provocada pela covid-19, que promoveu o teletrabalho e com ele levantou novas questões em torno da saúde docente. Assim, o exercício foi realizado em torno de três perguntas: $\mathrm{O}$ que dizem as pesquisas sobre a saúde/doença dos professores em tempos de covid-19? Como os docentes vivem a profissão no contexto da pandemia? E que experiências podem orientar a elaboração de políticas sociais para o bem-estar dos professores em tempos de incerteza? O trabalho foi elaborado a partir da sociologia dos corpos/emoções como perspectiva analítica e da revisão bibliográfica sistematizada como perspectiva metodológica. A revisão dos 26 artigos implicou a realização de quatro atividades: busca, avaliação,
Keywords: teacher, pandemic, health policy, health, mental health.

Palavraschave: docente, pandemia, política da saúde, saúde, saúde mental. 
análise e síntese. Conclui-se com o estudo que a saúde/doença docente é um tema recorrente nas pesquisas internacionais. $\mathrm{O}$ teletrabalho, como uma política educacional, perturbou a vida pessoal, profissional e familiar dos professores com efeitos sobre seu corpo e suas emoções. A evidência mostra uma diversidade de intervenções que podem ser consideradas na concepção de políticas para a classe docente em favor de uma saúde integral em tempos de pandemia. 


\section{Introducción}

En el siglo XXI, la docencia representa una profesión que se configura entre exigencias diversas, problemas sociales, parámetros de desempeño y procesos de evaluación. En diferentes regiones del mundo, ser docente alude a nuevas complejidades sociales, personales y físicas que, en conjunto, develan la precariedad de las condiciones de trabajo. Actualmente las políticas docentes - definidas como "las regulaciones y principios de actuación en el ámbito escolar y de sistemas educativos que dan forma, en un momento y lugar específicos, a los cuerpos docentes y sus acciones" (Organización para la Cooperación y el Desarrollo Económicos [OCDE], 2018, p. 4) -, se distinguen por el aumento de las tareas administrativas, el incremento de las presiones para mejorar el trabajo en el aula y el deterioro de la salud mental (Day, 2019). A nivel internacional, la docencia se vincula con problemas de salud general que incluye desde enfermedades estacionales hasta afecciones crónicas resultado de la carga ergonómica - disfonía, alteraciones musculoesqueléticas-; la sobrecarga laboral —estrés, insomnio, angustia-; y la invasión del espacio personal (Parra, 2005). En consecuencia, se reclama hacer de la salud docente un tema de política educativa a fin de generar intervenciones integrales (Robalino y Körner, 2005).

En marzo del 2020, el profesorado se enfrentó a un inédito y sorpresivo giro provocado por la pandemia del COVID-19. De un momento a otro, 165 países decretaron la vigilancia sanitaria, el distanciamiento social y el cierre de escuelas, afectando a 63 millones de docentes de educación primaria y secundaria (Organización de las Naciones Unidas para la Educación, la Ciencia y la Cultura [UNESCO], 2020). Las noticias acerca de la proliferación del virus, el aumento de nuevos casos y la obligatoriedad del confinamiento generaron una atmósfera de miedo y alarma, convirtiendo en prioridad el tema de la salud de la población en general, y de estudiantes y docentes, en particular (Stachteas y Stachteas, 2020; Trinidad, 2021). Pronto, la evidencia demostró sus efectos en la salud de las personas, en especial, el aumento del estrés psicológico (Kong et al., 2020).

En este contexto, la respuesta política fue imponer el teletrabajo docente de emergencia (Meo y Dabenigno, 2021), como estrategia para la continuidad pedagógica mediada por el uso de las tecnologías, lo que representó para el profesorado "implementar un trabajo 
de emergencia a distancia, sin contar con condiciones de trabajo y conectividad adecuados, sin capacitaciones previas, con horarios de trabajo extendidos, y sin una anticipación, orientaciones o un diseño claro desde el Ministerio de Educación" (Cornejo et al., 2020, párr. 3). Así, a la precarización histórica de la docencia se sumaron nuevas condiciones - largas jornadas laborales, altas exigencias, falta de valoración social-derivadas de la COVID-19 (Abanades, 2020; Pinheiro Almeida et al., 2021).

A nivel internacional, la salud docente se constituyó como objeto de estudio desde 1949 - a partir del trabajo en Estados Unidos de Cruze (1949), quien analizó la salud mental del profesorado encontrando que los sentimientos de inseguridad e inferioridad manifestados por los docentes de la época eran resultado del trabajo en el aula-; mientras, en Latinoamérica, desde la década de los setenta, cuando se reconocía la salud mental del profesorado como un problema serio (Parra, 2005; Sieglin y Ramos, 2007). Con todo, el conocimiento reciente da cuenta del incremento de las enfermedades físicas, mentales y sociales vinculadas a la docencia (Robalino, 2012).

Hoy, la COVID-19 emerge como campo de estudio en la investigación educativa internacional. Los estudios iniciales abordan, en distintas regiones y con diversos enfoques, el uso de la tecnología, el aprendizaje de los estudiantes -en particular de los que viven en pobreza - y los planes de reapertura de escuelas (Trinidad, 2021). En este sentido, el presente trabajo tiene como objetivo explorar los resultados de la investigación actual acerca de la salud docente, a fin de orientar la construcción de políticas sociales que contribuyan al bienestar del profesorado en tiempos de emergencia sanitaria. El ejercicio se realizó en torno a tres preguntas: ¿qué dice la investigación en cuanto a la salud/enfermedad ${ }^{1}$ del profesorado en tiempos de COVID-19?, ¿cómo viven los docentes la profesión en el contexto de pandemia?, y ¿qué experiencias pueden orientar el diseño de políticas sociales para el bienestar docente en momentos de incertidumbre? Conviene señalar que este estudio no pretende hacer una crítica detallada de las polí-

1 El empleo de la barra (/) se realiza, en apego a la propuesta de Scribano (2012), para "mostrar la separación/unión, distancia/proximidad y posibilidad/imposibilidad entre objetos/discursos que le otorgamos a lo que ha sido pensado como subcampos disciplinares separados, específicos y distantes" (p. 94). 
ticas docentes ante la COVID-19, sino documentar los aprendizajes construidos en torno a la salud docente como asunto prioritario en la agenda educativa.

Este texto forma parte de un proyecto de investigación en curso que gira en torno a la salud docente del profesorado de educación básica en el norte de México, con base en los siguientes supuestos:

- El deterioro del bienestar docente incide en la capacidad para realizar la labor pedagógica.

- Dado que el trabajo docente está mediado por las condiciones del contexto es probable que problemáticas asociadas a la pobreza, la desigualdad y la violencia trastoquen la forma en que los docentes asumen su trabajo.

- La formación docente constituye una estrategia clave en la prevención de enfermedades y la promoción de la salud entre el profesorado.

En particular, el presente trabajo se realiza desde la sociología de los cuerpos/emociones como perspectiva analítica. Con base en Scribano (2012), se asume que, en la investigación acerca de la salud docente "no es posible indagar y reflexionar sobre cuerpos/emociones por separado (...) [sino como] experiencia que anude cuerpo, emociones, acción colectiva, conflicto y producción ideológica" (pp. 94-95). Se considera que en el estudio de los cuerpos/corporalidades de los docentes se cruzan procesos diferenciados de salud-enfermedadatención mediados por los afectos/emociones.

\section{Metodología}

El ejercicio cualitativo se realizó con base en los principios de la revisión bibliográfica sistematizada consistente en una investigación bibliográfica en torno a un tópico concreto, a través del cumplimiento de un conjunto de aspectos - selección de fuentes, procedimiento de búsqueda (fecha y descriptores), criterios de inclusión/exclusión, esquemas de análisis, procedimientos para la síntesis y presentación de resultados-que permiten que la información sea revisada, depurada y actualizada (Codina, 2020).

Dado que las revisiones sistemáticas parten de preguntas de investigación, el ejercicio tomó como guía las cuestiones expuestas en la intro- 
ducción. La base de la evidencia se conformó, inicialmente, por 82 artículos en torno a la salud docente publicados en revistas científicas que se localizaron en diferentes bases de datos como Elton Bryson Stephens Company (EBSCO), la Red de Revistas Científicas de América Latina y el Caribe, España y Portugal (Redalyc) y la Scientific Electronic Library Online (SciELO) entre el año 2002 y 2021. En atención al interés del presente texto, la búsqueda se redefinió con los términos "salud", "docente" y "COVID-19", conectados por el operador booleano and, que fueron utilizados como descriptores en español e inglés. Se consideraron tres criterios:

- El temporal: se incluyeron trabajos publicados durante el último año (2020-2021).

- El espacial: se examinaron aquellos vinculados con el profesorado en diversas regiones del mundo - Argentina (Meo y Dabenigno, 2021); Brasil (Pinheiro Almeida et al., 2021); Chile (Bravo et al., 2020; Ramos-Huenteo et al., 2020; Rojas et al, 2020); Colombia (Burbano et al., 2020; Gañán et al., 2020; Inciarte et al., 2020; Prada et al., 2021); Costa Rica (Sibaja et al., 2020); España (Abanades, 2020; Aperribai et al., 2020; Ozamiz-Etxebarria et al., 2021a; Ozamiz-Etxebarria et al., 2021b), Estados Unidos (Roman, 2020; Trinidad, 2021); Fiyi (Dayal y Tiko, 2020); Italia (Matiz et al., 2020); Grecia (Stachteas y Stachteas, 2020), México (Sánchez et al., 2020); y Turquía (Çifçi y Demir, 2020; Karakaya et al., 2021; Kayabınar et al., 2020).

- El temático: la totalidad de los artículos abordan la salud docente en tiempos de COVID-19 como tema central.

La depuración posterior de los resultados se realizó en atención a lo siguiente:

- Criterio de inclusión: se admitieron aquellos artículos con referencia a la salud del profesorado en diversas regiones y niveles educativos -educación básica (Aperribai et al., 2020; Bravo et al., 2020; Dayal y Tiko, 2020; Inciarte et al., 2020; Kayabınar et al., 2020; Matiz et al., 2020; Meo y Dabenigno, 2021; Pinheiro Almeida et al., 2021; Stachteas y Stachteas, 2020); educación media (Inciarte et al., 2020; Karakaya et al., 2021; Pinheiro Almeida et al., 2021); educación superior (Abanades, 2020; Burbano et al., 2020; Casimiro et al., 2020; Gañán et al., 2020; Ozamiz-Etxebarria et al., 2021a; Prada et al., 2021; Sánchez et al., 2020; Sibaja et al., 2020). 
- Criterio de exclusión: se descartaron los artículos publicados antes del año 2020, escritos en idiomas distintos del castellano, inglés y portugués, cartas al editor y ensayos, además de aquellos sin referencia a la emergencia sanitaria.

El proceso se realizó en las cuatro etapas propuestas por Grant y Booth (2009): búsqueda, evaluación, análisis y síntesis.

Tabla 1

Etapas para la revisión bibliográfica sistematizada

\begin{tabular}{|c|c|c|}
\hline Búsqueda & $\begin{array}{l}\text { Conformación de la base de la evidencia o ban } \\
\text { documentos (EBSCO, Redalyc y SciELO) }\end{array}$ & co de \\
\hline Evaluación & $\begin{array}{l}\text { Valoración inicial de los documentos } \\
\text { Tema central y temáticas tangenciales/ } \\
\text { estructura de los artículos científicos. } \\
\text { Criterios de inclusión/exclusión } \\
\text { Preguntas guía: ¿Se identifica en el } \\
\text { documento alguna relación entre salud y } \\
\text { docentes/profesores? }\end{array}$ & 82 artículos \\
\hline Análisis & $\begin{array}{l}\text { Depuración de los documentos } \\
\text { Se incluyen los artículos sobre salud, docente, } \\
\text { COVID-19. } \\
\text { Preguntas guía: ¿Se identifica en el } \\
\text { documento alguna relación entre salud, } \\
\text { docentes y COVID-19? }\end{array}$ & 26 artículos \\
\hline Síntesis & Presentación de los resultados del análisis en te & mas centrales \\
\hline
\end{tabular}

Fuente: Elaboración propia sobre la base de Codina (2020) y Grant y Booth (2009).

El análisis de los 26 artículos se realizó a partir del modelo interactivo propuesto por Miles et al. (2014) definido como un proceso cíclico en el que interactúan tres actividades:

- Condensación de datos: se realizó a través de una matriz de análisis cualitativo que permitió recuperar el material más significativo, reunir fragmentos de datos y resumir la información.

- Presentación de datos: para el ensamble de la información se construyó un sistema de categorías/subcategorías predeterminadas y emergentes (Tabla 2).

- Elaboración/verificación de conclusiones: se realizó mediante síntesis narrativas por cada una de las categorías de análisis. 
Tabla 2

Representación del sistema de categorías y subcategorías de análisis

\begin{tabular}{|c|c|c|c|c|c|}
\hline \multirow{5}{*}{ 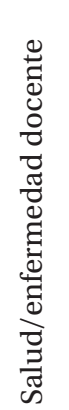 } & \multirow{3}{*}{$\begin{array}{l}\text { Salud/ } \\
\text { enfermedad } \\
\text { física Cuerpo }\end{array}$} & \multirow{5}{*}{ 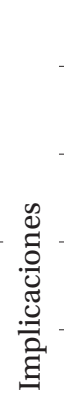 } & Teletrabajo & \multirow{5}{*}{ 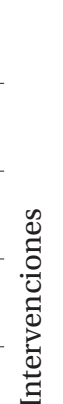 } & $\begin{array}{l}\text { Continuidad } \\
\text { académica }\end{array}$ \\
\hline & & & Vida personal & & $\begin{array}{l}\text { Formación } \\
\text { docente }\end{array}$ \\
\hline & & & Vida familiar & & $\begin{array}{l}\text { Equipamiento y } \\
\text { conectividad }\end{array}$ \\
\hline & $\begin{array}{l}\text { Salud/ } \\
\text { enfermedad }\end{array}$ & & $\begin{array}{l}\text { Vida } \\
\text { profesional }\end{array}$ & & Intervenciones \\
\hline & $\begin{array}{l}\text { mental } \\
\text { Emociones }\end{array}$ & & Respuestas & & Autocuidado \\
\hline
\end{tabular}

\section{Resultados}

En esta sección se presentan los resultados del análisis cualitativo con fundamento en las preguntas de investigación: la salud/enfermedad del profesorado en tiempos de COVID-19; las implicaciones de la pandemia en la vida de los docentes; y las intervenciones realizadas para atender el bienestar docente en momentos de incertidumbre.

Salud/enfermedad docente: aproximaciones desde la investigación

En términos generales, la salud alude al "estado de completo bienestar físico, mental y social, y no solamente la ausencia de afecciones o enfermedades (...) [constituye] uno de los derechos fundamentales de todo ser humano" (Organización Mundial de la Salud [OMS], 2019, párr. 1). En esta definición se reconocen tres puntos clave: la condición de bienestar, el equilibrio entre lo físico, mental y social, y el papel del contexto. De este modo, el estado de salud/enfermedad depende tanto de factores biológicos como de aspectos ambientales, psicológicos y sociales; se asume así, que el binomio salud/enfermedad es un proceso que requiere explicarse en su determinación social e histórica. Además, desde la sociología de los cuerpos/emociones, el análisis de la salud/enfermedad, en tanto construcción social, remite a "cuerpos [que] se observan situados en emociones" (Scribano, 2012, p. 99). 
Según la evidencia, la COVID-19 afectó de múltiples formas la vida de los docentes, en especial, su salud física, mental y social (Bravo et al., 2020; Pinheiro Almeida et al., 2021; Sahu, 2020). La suma de factores externos (aumento de la mortalidad, incertidumbre, problemas económicos, imposición de la modalidad virtual, sobrecarga laboral, ampliación de la jornada, descalificación del trabajo por parte del personal directivo, falta de contacto personal, contextos de fuerte desigualdad y precarias condiciones materiales de trabajo), o internos (sentimientos de indignación, falta de conectividad, débil organización del tiempo, condiciones del espacio y distracciones del hogar), entre otros provocaron cambios significativos en la vida magisterial en forma de bienestar/malestar (Aperribai et al., 2020; Gañán et al., 2020; Karakaya et al., 2021; Meo y Dabenigno, 2021). El exceso de trabajo implicó mayor cantidad de tiempo para atender los compromisos laborales desde casa, lo cual derivó en el aumento del cansancio crónico, en la reducción del descanso durante la jornada laboral y la dependencia de terceros. Esta intensificación del trabajo docente constituye "una de las formas más tangibles mediante la cual los educadores son degradados en su condición profesional y social" (Bravo et al., 2020, p. 1004).

La COVID-19 trastocó no solo los aspectos biofisiológicos de los docentes - huellas sociales en la biología de los cuerpos/emociones y viceversa-, sino sus biografías (escrituras sociales donde lo social se hace carne incorporando estados de sensibilidades en los cuerpos/emociones) (Scribano, 2012). Con la pandemia, la salud física del profesorado se deterioró. El cuerpo, en tanto condición material de la existencia, encarnó el dolor y la enfermedad. Previamente a la llegada de la COVID-19 los problemas de salud/enfermedad asociados a exigencias ergonómicas y afecciones crónicas estaban presentes en la vida de los maestros latinoamericanos (Parra, 2005), como resultado de estilos de vida sedentarios, ausencia de tiempos y espacios para la alimentación saludable. Luego de la pandemia, las enfermedades ergonómicas se agudizaron: del dolor en las extremidades inferiores por el trabajo de pie frente a la pizarra, y en las extremidades superiores debido a las constantes actividades de escritura - asociadas a la permanencia de metodologías frontales y poco participativas (Robalino y Körner, 2005) -, los docentes se enfrentaron a posturas prolongadas frente a la computadora, sin estaciones de 
trabajo adecuadas, que incrementaron la fatiga ocular y la prevalencia, intensidad y frecuencia de los problemas musculoesqueléticos en forma de dolor en cuello, espalda, zona lumbar y articulaciones de la cadera (Aperribai et al., 2020; Kayabınar et al., 2020).

Pero la COVID-19 no solo afectó los cuerpos de los docentes, sino también sus emociones (Abanades, 2020; Casimiro et al., 2020; Dayal y Tiko, 2020; Meo y Dabenigno, 2021; Ramos-Huenteo et al., 2020; Sánchez et al., 2020). En medio del confinamiento, la docencia se gestó en

un cóctel de acciones y emociones (...) [ante] la profunda incertidumbre, consecuencia de no saber cómo avanzará la pandemia ..., de no saber si nosotros o nuestros seres queridos seremos víctimas de la infección y sus complicaciones, de la ausencia de una vacuna y tratamiento específico, y de la falta de certeza de cuándo regresaremos a nuestras actividades cotidianas. (Sánchez et al., 2020, p. 3).

Respecto de la salud mental, Kayabınar et al. (2020), tomando como referencia a un grupo de docentes de educación primaria y secundaria en Turquía, encontraron que, independientemente de si los profesores imparten educación en línea o no, la pandemia per se puede aumentar los niveles de ansiedad y depresión ante el miedo de enfermar o morir. En esta línea, diversos estudios demuestran que niveles altos de estrés, angustia y ansiedad, depresión, tristeza, cansancio, reducción de la concentración, falta de motivación, alteraciones o trastornos del sueño, ataques de pánico, miedo a enfermarse, perder el trabajo y preocupaciones relacionadas con los deberes profesionales, afectaron de modo considerable la salud mental del profesorado (Aperribai et al., 2020; Casimiro et al., 2020; Dayal y Tiko, 2020; Kayabınar et al., 2020; Meo y Dabenigno, 2021; Ozamiz-Etxebarria et al., 2021a; Ozamiz-Etxebarria et al., 2021b; Prada et al., 2021; Robinet-Serrano y Pérez-Azahuanche, 2020; Stachteas y Stachteas, 2020; Trinidad, 2021). Para Gañán et al. (2020), a partir del estudio realizado con docentes universitarios en Colombia, se trata de tecnoestrés laboral, entendido como el "estado psicológico negativo que se relaciona con la percepción de un desajuste entre las demandas y los recursos relacionados con el uso de las TIC" (p. 3). En el caso de los docentes universitarios en Latinoamérica, la evidencia señala un estado emocional bajo, resultado del aumento significativo en los niveles de estrés, ansiedad y angustia (Casimiro et al., 2020). 
En este punto, destaca la situación de las profesoras quienes en América Latina (Casimiro et al., 2020), en Italia (Matiz et al., 2020), en España (Ozamiz-Etxebarria et al., 2021a), y en Grecia (Stachteas y Stachteas, 2020), presentan mayor incidencia. En el caso de la docencia universitaria, las mujeres en el grupo de 44 a 54 años tienen mayor riesgo, dada la doble tarea que ellas realizan: por un lado, el trabajo a distancia y, por el otro, el cuidado de la familia y el hogar (Casimiro et al., 2020). En el contexto italiano, la COVID-19 y las medidas de salud pública resultaron en un impacto negativo en la salud mental de las profesoras que presentaron un mayor riesgo a desarrollar síntomas de estrés, ansiedad y depresión (Matiz et al., 2020). Este hallazgo resulta clave para México, en donde las mujeres representan el 86,8\% del profesorado en educación preescolar, el 64,4\% en educación primaria y el 53,5\% en educación secundaria (Comisión Nacional para la Mejora Continua de la Educación [MEJOREDU], 2020). Paradójicamente, Prada et al. (2021) concluyeron que los profesores de una universidad pública en Colombia tienden a sufrir mayores señales de depresión, posiblemente por el cambio brusco en los hábitos de vida. Por su parte, Ozamiz-Etxebarria et al. (2021a) encontraron un incremento en los niveles de ansiedad, depresión y estrés entre el profesorado, particularmente en personas mayores, de educación preescolar y primaria, así como aquellos con inestabilidad laboral.

En el marco de la crisis por la COVID-19, docentes con nombramiento interino o provisional, con contratos temporales, bajo figuras de docentes sustitutos y personal de apoyo educativo, temen la finalización de sus contratos y la pérdida de su salario. En esta línea, conservar el empleo y los salarios constituye una de las recomendaciones de la UNESCO (2020) para los Estados, en tanto,

esta crisis no puede ser un pretexto para (...) dejar a un lado los derechos laborales. Los gobiernos y todos los proveedores de educación, tanto pública como privada, deben trabajar para preservar a todo el personal educativo, sus salarios y beneficios. (pp. 1-2).

\section{COVID-19. Implicaciones en la vida docente}

Ante la llegada de la COVID-19, el trabajo docente convulsionó. A partir de marzo de 2020, la docencia asumió las características del teletrabajo, que se caracteriza por la ampliación de la jornada laboral, el 
dominio de nuevas habilidades y la falta de contacto físico (Aperribai et al., 2020; Burbano et al., 2020; Gañán et al., 2020; Inciarte et al., 2020; Meo y Dabenigno, 2021; Ramos-Huenteo et al., 2020). Para Meo y Dabenigno (2021), en el fondo, se trata de teletrabajo forzado en el hogar:

una forma de teletrabajo porque implica el uso de TIC para la realización remota de aspectos centrales del trabajo, el cual se desarrolla en los hogares de los/as trabajadores y es puesto en marcha sin la expresa voluntad de las partes involucradas (empleados/as ni empleadores). (p. 104).

Los primeros efectos de la pandemia se mostraron en el ámbito personal y familiar (Aperribai et al., 2020; Bravo et al., 2020; Burbano et al., 2020; Kayabınar et al., 2020; Meo y Dabenigno, 2021; Sánchez et al., 2020). En la esfera personal, el profesorado precisó modificar el estilo de vida habitual, la rutina diaria, el presupuesto - reorganizado para la compra de equipo de cómputo y planes de servicio de Internet-, el espacio íntimo - antes dedicado al descanso y la recreación ahora convertido en oficina móvil-; mientras, en el ámbito familiar, los docentes aluden a una alteración del genio/corrosión del carácter, así como enfrentar dificultades para conciliar el tiempo destinado a las responsabilidades domésticas y de cuidado, develando el deterioro del equilibrio entre la vida laboral y personal de los docentes durante el confinamiento.

En la docencia entre pantallas, la imagen del profesorado se resguarda al apagar la cámara "porque es pudoroso mostrar los espacios, que inevitablemente mostrará su vulnerabilidad" (Bravo et al., 2020, p. 1006). De este modo, la salud/enfermedad del profesorado se vincula directamente con el cuerpo imagen, que alude a las posibilidades de presentación social de la persona y el reconocimiento social del gremio; con el cuerpo piel, que refiere a las potencialidades para experimentar/se en el mundo; y con el cuerpo movimiento, que apunta a las capacidades para desplazarse/hacer en el mundo social más allá del confinamiento y la distancia física (Scribano, 2012).

En el ámbito profesional, los docentes desafiaron dificultades de carácter logístico, vinculadas con el manejo del tiempo, horarios de clase, espacios físicos para el trabajo a distancia; tecnológico, relacionadas al acceso a Internet, disposición de equipos de cómputo, conocimien- 
tos de plataformas educativas; pedagógico, ligadas al conocimiento de herramientas didácticas virtuales, manejo de grupos a distancia, evaluación remota de los aprendizajes; y socioafectivo, afines a aspectos emocionales derivados de sentimientos de tristeza, frustración, ansiedad y cansancio (Sánchez et al., 2020). Además, los maestros se enfrentaron a la presión y vigilancia por parte de las autoridades educativas. La cultura escolar, las relaciones sociales de trabajo, el liderazgo de los directores y la participación del profesorado en la toma de decisiones escolares, aparecen como temas recurrentes en los estudios relativos a la salud docente (Robalino y Körner, 2005). En la escuela - como espacio físico o virtual de trabajo-, la docencia se configura en una red de interacciones (individuales, grupales, contextuales y sociales), jerárquicas y horizontales que denotan la complejidad del trabajo docente (Parra, 2005); en ella, se desarrollan condiciones materiales y sociales que pueden contribuir al bienestar/malestar del profesorado (Robalino, 2012). Así, una cultura escolar que impulsa relaciones sociales de participación social, cordialidad, cercanía y confianza entre sus miembros constituye una fuente de satisfacción laboral. En sentido contrario, culturas institucionales restrictivas, autoritarias y fiscalizadoras impactan negativamente la salud mental de los docentes (Meo y Dabenigno, 2021).

En épocas de pandemia son pertinentes las culturas escolares flexibles y sensibles con capacidad para generar climas de trabajo que garanticen el logro de los aprendizajes sin detrimento de la salud mental del profesorado. En este sentido, destacan el liderazgo del personal directivo y la capacidad de las escuelas para tomar decisiones en periodos de emergencia (Rojas et al., 2020; Trinidad, 2021). Según el trabajo realizado por Rojas et al. (2020) en Chile, frente a la COVID-19, los directores escolares podían implementar estrategias para producir mejoras en las prácticas educativas, realizar acciones para que los docentes hicieran suyo el sentido del cambio, impulsar el trabajo colaborativo entre el colectivo, aprovechar las habilidades y destrezas de su personal, entre otras; sin embargo, los resultados de su estudio mostraron que la dirección escolar no articuló los recursos materiales y humanos para el adecuado funcionamiento de la escuela frente a la emergencia, generando estrés y agotamiento emocional entre el profesorado. Para Trinidad (2021), con base en una muestra representativa de docentes 
y líderes escolares de EE.UU., centrar el análisis en la toma de decisiones en momentos de crisis, las preocupaciones de los docentes y las prioridades de los directores escolares, así como sus consecuencias, representa una nueva línea de investigación que debe cultivarse.

Las respuestas del profesorado ante la imposición del teletrabajo docente fueron diversas:

- de resistencia (Gañán et al., 2020; Pinheiro Almeida et al., 2021);

- de resiliencia, entendida como la capacidad para prosperar ante la adversidad (Burbano et al., 2020; Casimiro et al., 2020; Matiz et al., 2020; Robinet-Serrano y Pérez-Azahuanche, 2020);

- de adaptación, a través de procesos de familiarización y habituación ante el tecnoestrés laboral (Gañán et al., 2020);

- de afrontamiento, mediante diferentes estrategias de defensa (Gañán et al., 2020; Robinet-Serrano y Pérez-Azahuanche, 2020).

En este punto, Dayal y Tiko (2020) en Fiyi, Karakaya et al. (2021) en Turquía y Sibaja et al. (2020) en Costa Rica, encontraron actitudes positivas entre el profesorado. El optimismo, el compromiso con el estudiantado y la oportunidad de aprender fueron claves en el manejo de la crisis entre algunos docentes que buscaron formas novedosas de enseñanza, convirtiendo la pandemia en una posibilidad para fortalecer sus competencias tecnológicas, comunicativas y socioemocionales. Para Sibaja et al. (2020) la COVID-19 representó una oportunidad para reinventar la práctica docente del profesorado costarricense. En opinión del estudiantado español, una buena docencia en momentos de incertidumbre es aquella que se distingue por habilidades de comunicación, como la escucha activa, actitudes resilientes e inteligencia emocional (Abanades, 2020).

La recreación representa un componente clave para mantener la buena salud física y mental. Pese a su importancia, la evidencia indica que los docentes han postergado el uso del tiempo libre dedicado usualmente al descanso y al disfrute (Robalino y Körner, 2005; Sánchez et al., 2020). Previamente a la pandemia, los docentes encontraban satisfacción en la lectura, los paseos familiares, las reuniones con amistades y en la participación en cursos de desarrollo personal (Robalino y Körner, 2005). El confinamiento sanitario obligó al profesorado a buscar nuevas formas de sobrellevar el encierro. Para Gañán 
et al. (2020) las actividades recreativas se convirtieron en estrategias de afrontamiento de dos tipos: individuales como dormir, leer, cocinar y ejercitarse; y colectivas, como los espacios de formación docente. En este caso, sobresalen las iniciativas generadas por las propias instituciones dirigidas a contener el desánimo, por ejemplo, las capacitaciones en diversos temas generadas por una universidad en Colombia (Burbano et al., 2020).

La evidencia internacional demuestra que los docentes han implementado, por iniciativa propia, rápidas e innovadoras respuestas frente a la emergencia sanitaria, por lo que se recomienda capitalizar su experiencia y habilidad en el desarrollo de acciones educativas frente a la COVID-19. Sus voces constituyen un referente central para valorar el éxito de las acciones implementadas en torno a la evaluación del aprendizaje, el desarrollo curricular, el ajuste de los calendarios escolares y la pertinencia de las plataformas virtuales, entre otros (UNESCO, 2020).

\section{Acciones para el bienestar docente en tiempos de emergencia sanitaria}

De forma sucesiva, las acciones de política educativa y sanitaria implicaron: la declaración del estado de emergencia que se tradujo en la suspensión de las actividades presenciales y el cierre de escuelas; la implementación de estrategias para la continuidad académica mediante la imposición del trabajo docente en casa, la capacitación docente en el uso de las TIC, la flexibilización del calendario escolar, la extensión del horario laboral y el diseño de materiales educativos para garantizar las oportunidades de aprendizaje a distancia; el incremento de acceso a Internet y la dotación de equipo de cómputo (Inciarte et al., 2020). En conjunto, la respuesta gubernamental para el profesorado se centró en la política de continuidad académica a través de las TIC, que tuvo dos efectos inmediatos: primero, afectó el trabajo docente: de modo repentino, los docentes en el mundo "se vieron obligados/as a usar TIC en sus interacciones con autoridades, docentes, adolescentes y familias, y a hacerlo desde sus hogares con sus propios recursos tecnológicos, en tiempos y espacios ajenos a lo escolar" (Meo y Dabenigno, 2021, p. 104); y segundo, visibilizó la desigualdad. 
En la experiencia docente, las condiciones de apropiación diferencial y desigual de bienes comunes determinaron el éxito de los programas de continuidad académica y construyeron/modelaron las interrelaciones/correspondencias entre cuerpos/emociones (Inciarte et al., 2020; Scribano, 2012). Por ejemplo, el estudio realizado en ciertos territorios de la Argentina demostró que los docentes con ventaja económica, que laboraban en escuelas privadas, con alumnado de nivel socioeconómico alto, en residencias sin menores y niveles de responsabilidad doméstica y de cuidado limitado respondieron mejor a las exigencias de la educación a distancia (Meo y Dabenigno, 2021). De forma complementaria, la COVID-19 visibilizó la incapacidad, precarización y obsolescencia de los sistemas públicos de salud (Sánchez et al., 2020; UNESCO, 2020), situación que hace necesario "aprender las lecciones de otros países (...) para prevenir y atender a la población acerca de la pandemia por COVID-19, y al manejo de estrategias y políticas de salud pública" (Sánchez et al., 2020). En el contexto actual, la salud como proceso social "señala directamente hacia los cruces y entramados entre cuerpos/emociones dado su carácter de construcción social, atravesada por las consecuencias de las políticas de los cuerpos y las políticas de las emociones que las sociedades elaboran y practican" (Scribano, 2012, p. 104).

\section{Formación docente}

Dado que las políticas educativas priorizaron el uso de las TIC, sin considerar la preparación y las herramientas del profesorado para impartir educación en línea (Kayabınar et al., 2020), pronto la mayoría de los docentes reconoció la carencia de conocimientos y habilidades en el área (Inciarte et al., 2020; Meo y Dabenigno, 2021; Stachteas y Stachteas, 2020), salvo algunos profesores de escuelas privadas que contaban con experiencia previa alrededor de las TIC (Meo y Dabenigno, 2021). En respuesta, organismos internacionales se pronunciaron por proveer a los docentes apoyo profesional y entrenamiento adecuados (UNESCO, 2020). Así, se generaron diversas estrategias para fortalecer las competencias digitales de los docentes, entendidas como el conjunto de conocimientos, habilidades y actitudes para hacer un uso consciente, seguro, creativo y crítico de las TIC en el trabajo, el ocio y la comunicación (Abanades, 2020; Aperribai et al., 2020; Karakaya et al., 2021; Pérez et al., 2020). En este rubro, se requieren de itinerarios for- 
mativos diversificados según la trayectoria de los docentes, dado que la evidencia demuestra una asociación moderada entre la edad y los niveles de competencia digital (Burbano et al., 2020). Por su parte, el trabajo de Meo y Dabenigno (2021) da cuenta del acompañamiento pedagógico por parte de pares y de la dirección escolar.

Además, las necesidades de formación docente expusieron la urgencia de trayectos formativos para consolidar la comunicación efectiva a través de estrategias para vivir la incertidumbre y dar respuesta a la crisis, revelando el apremio por atender la salud emocional entre el profesorado (Inciarte et al., 2020; Roman, 2020). Priorizar la salud y el bienestar de los docentes, en especial en aquellas regiones donde el riesgo por COVID-19 está en constante aumento, representa un reto para los gobiernos, particularmente, porque la salud mental del profesorado constituye un tabú dentro de las políticas educativas (Sieglin y Ramos, 2007; UNESCO, 2020). En este punto, destaca la necesidad de incluir la salud docente como un componente curricular en los programas de formación inicial y formación continua. Previamente a la pandemia, Robalino (2012) y Zamora-Díaz et al. (2017) coincidían en la importancia de formar a los docentes para reflexionar acerca de la salud y su protección, en especial, al profesorado principiante; frente a la COVID-19, se propone dar un giro a la formación docente: de la prevención se interpela al profesorado a capacitarse para brindar apoyo a las personas afectadas por la crisis sanitaria (Roman, 2020).

\section{Equipamiento y conectividad}

Según la evidencia, la implementación de los programas de continuidad académica, fueron eficientes solo para docentes, estudiantes y familias con electricidad adecuada, conexión a Internet, computadoras y espacios físicos para el trabajo. De esta manera, las posibilidades de equipamiento y acceso a Internet visibilizaron las condiciones de desigualdad en que vive una parte significativa del profesorado, especialmente, de aquellos que trabajan en áreas rurales o en comunidades urbanas con bajos recursos o vulnerabilidad (UNESCO, 2020).

Al respecto, Meo y Dabenigno (2021) encontraron que solo la mitad de los docentes de algunas regiones de Argentina contaba con espacio aislado y acceso a computadora de uso exclusivo; mientras, Inciarte et al. (2020) documentan la contratación de planes pospago para el uso 
de Internet, así como el otorgamiento de computadoras a las escuelas participantes en el programa Computadores para Educar (CPE) implementado en Colombia. En México, durante el ciclo 2018-2019, únicamente el 34,6\% de las escuelas primarias, el 45,3\% de las secundarias y 44,1\% de las de educación media superior contaban con conexión a Internet. Ante esta situación, "se espera que el reconocimiento del acceso a Internet como un derecho humano plasmado en la Constitución pueda aumentar el porcentaje de personas con este servicio" (MEJOREDU, 2020, p. 66).

Además, la carencia de equipo de cómputo, el aumento del gasto personal/familiar en la adquisición de alguno, su uso compartido al interior de las familias, la conexión limitada o intermitente incrementaron los sentimientos de angustia, estrés y frustración entre el profesorado (Cornejo et al., 2020; Gañán et al., 2020; Rojas et al., 2020; Sibaja et al., 2020).

\section{Intervenciones}

El análisis de la producción muestra un conjunto de iniciativas que se caracterizan por transitar de la reflexión a la acción en torno a la salud docente. Tradicionalmente, la docencia se vincula con una vida sedentaria que compromete la calidad vital del profesorado (Burbano et al., 2020; Robalino y Körner, 2005). Una rutina de vida sin espacios para la ejercitación corporal atenta contra la salud física y mental de los docentes. En respuesta, la actividad física constituye un factor central en la prevención de trastornos mentales como la ansiedad o la depresión, particularmente, en situaciones de encierro (Aperribai et al., 2020; Çifçi y Demir, 2020). Ante la COVID-19, la actividad física en casa resultó una estrategia exitosa para prevenir problemas de salud entre los docentes de educación primaria y secundaria en España y el profesorado en servicio y en formación en Turquía, lo que puede impulsarse en emergencias similares.

En el diseño de nuevos modelos de cuidado de la salud a la distancia se propone la incorporación de las TIC (Kayabınar et al., 2020; Roman, 2020). En el rubro de la prevención, Kayabınar et al. (2020) proponen la telerrehabilitación para atender los problemas musculoesqueléticos y psicosociales entre los docentes, en especial, entre aquellos que carecen de oportunidades de fisioterapia cara a cara. Por su parte, Roman 
(2020) da cuenta del uso de las herramientas de tecnología educativa para abordar las necesidades de salud emocional de un grupo de docentes en formación de una universidad en EE.UU. durante la pandemia. Así, la salud digital, la ciber salud o esalud constituyen alternativas asociadas al desarrollo y uso de las tecnologías digitales en la mejora de la salud (Pérez et al., 2020).

Kayabinar et al. (2020), Matiz et al. (2020), Ozamiz-Etxebarria et al. (2021a, 2021b), Prada et al. (2021) y, Robinet-Serrano y Pérez-Azahuanche (2020) coinciden en la importancia del apoyo psicosocial durante la pandemia, develando la importancia de las emociones, unidades de experienciación como "acción y efecto de sentir o sentirse (...) en el mundo" (Scribano, 2012, p. 102). Brindar atención psicológica al profesorado no solo les permitirá desempeñar mejor su función, sino garantizar el bienestar del estudiantado (Ozamiz-Etxebarria et al., 2021a, 2021b). Con este propósito, la Meditación Orientada a la Atención Plena (MOM, por sus siglas en inglés), talleres de yoga o los programas de mindfulness se recomiendan para reducir los efectos negativos en la salud mental por la COVID-19, en particular, para restaurar el bienestar de las personas más vulnerables (Matiz et al., 2020; Ozamiz-Etxebarria et al., 2021b). Por su parte, la revisión elaborada por Robinet-Serrano y Pérez-Azahuanche (2020) destaca los beneficios de las técnicas de relajación (aflojar los músculos, respirar profundamente, recrearse en imágenes agradables) que contribuyen a mitigar el impacto negativo del estrés.

Si bien la salud docente alude a un tema de política nacional que conmina la responsabilidad de los gobiernos, también refiere a un elemento de compromiso personal por parte del profesorado (Robalino y Körner, 2005; Roman, 2020; Sahu, 2020). En esta línea, las acciones de autocuidado y protección cobran relevancia, fundamentalmente, porque la identidad profesional se sostiene en narrativas que destacan el compromiso con el cuidado de los otros que, con frecuencia, se traduce en dificultades para reconocer las propias necesidades y el cuidado de sí mismo (Pinheiro Almeida et al., 2021; Trinidad, 2021). El autocuidado se propone como una estrategia clave en la promoción de la salud mental que debe ser considerada en el establecimiento de políticas de protección y el diseño de intervenciones para la atención integral, con énfasis en la sensibilización dado que, ante la ausencia 
de programas de salud mental es común que los docentes prefieran el silencio por miedo a ser juzgados o avergonzados (Pinheiro Almeida et al., 2021).

Finalmente, Stachteas y Stachteas (2020) centran la atención en la resiliencia, es decir, la capacidad para enfrentar la adversidad de forma constructiva. En situaciones de incertidumbre laboral, los docentes más resilientes logran mejores respuestas que devienen en la disminución del estrés crónico. Así, se anima el diseño de intervenciones que promuevan la resiliencia docente. Al respecto, Day (2019) define la resiliencia docente como un rasgo dinámico y latente, producto de la interacción diaria entre la historia personal y profesional que se fortalece en las relaciones recíprocas de apoyo entre colegas. Desde la noción de resiliencia del día a día, señala la relación directa entre las emociones positivas y la salud.

\section{Reflexiones finales}

El trabajo permitió, en primer lugar, identificar las principales líneas del debate académico acerca de las condiciones de la salud docente durante la pandemia a partir de los resultados de la investigación reciente en torno al tema. La suma de factores externos e internos provocaron cambios en la salud/enfermedad física y mental del profesorado en el escenario internacional. Por otra parte, el teletrabajo, como política educativa, trastocó la vida personal, profesional y familiar del profesorado. Estos cambios mostraron las dificultades para conciliar la vida personal y laboral de los docentes durante la pandemia, el papel clave del personal directivo en la construcción de culturas escolares en favor del trabajo colaborativo y la riqueza de la experiencia docente en momentos de crisis. Respecto de las acciones para el bienestar docente, la evidencia da cuenta de una diversidad de intervenciones que pueden ser consideradas en el diseño de políticas docentes en favor de su salud integral en tiempos de pandemia. A pesar de la pertinencia y relativo éxito en la disminución de los niveles de estrés, ansiedad y angustia, se trata de iniciativas particulares, aisladas y focalizadas que dependen de la voluntad de sus promotores y de la disponibilidad de recursos financieros y humanos que deberán considerarse tomando en cuenta las características de las poblaciones destinatarias (con/sin conectividad, grandes ciuda- 
des/pequeños poblados, contexto urbano/rural, niveles del sistema escolar, entre otras).

En el marco de las políticas docentes, la salud ocupó un lugar tangencial. Desde la posición de Scribano (2012) se trata de una política de los cuerpos legitimada por la sociedad que materializa la estructuración del poder. Los estudios recientes respecto de la salud docente confirman las formas de dominación sobre el magisterio: la precarización de las condiciones de trabajo y la desvalorización social de la docencia marcan la experiencia corporal/emocional del profesorado. En medio de la pandemia, las marcas corporales son evidentes en la salud/enfermedad física de los docentes, mientras la ausencia de acciones gubernamentales para atender la salud/enfermedad mental define la construcción de una sensibilidad social ligada a la apatía y la evitación sistemática del conflicto. El confinamiento - al impulsar la distancia física y limitar los vínculos sociales-, sumado a la precariedad del trabajo docente, promueve la indiferencia e impide la construcción de una narrativa compartida ante las dificultades de la emergencia sanitaria; asimismo, dificulta el desarrollo de cualidades de lealtad, compromiso institucional, reciprocidad, cuidado, dependencia y apoyos mutuos. En este sentido, el aislamiento condena "nuestras capacidades sociales, de confiablidad e importancia para los demás y, en definitiva, de la constancia personal sobre la que sostiene el carácter, transformando a las personas frágiles e irascibles" (Bravo et al., 2020, p. 1004). El sobrepeso - resultado del sedentarismo y la falta de actividad física-, el cansancio y una historia reciente de momentos de estrés y angustia como "marcas corporales son inscripciones socialmente establecidas por el proceso de dominación en el que está sumido [el profesorado]" (Scribano, 2012, p. 102). En tanto que "la docencia es un trabajo emocional" (Day, 2019, p. 68), se demuestra la necesidad de incorporar estrategias para atender la salud mental docente en las agendas de política educativa, sobre todo, porque la salud es responsabilidad de los gobiernos (OMS, 2019; Robalino y Körner, 2005).

La presente revisión muestra algunas limitaciones resultado del periodo de búsqueda, la novedad del tema abordado y el posible sesgo en la selección de los artículos, a partir de los criterios de inclusión/ exclusión considerados, particularmente, porque se descartaron las 
producciones que no transitan necesariamente por los circuitos académicos, pero que son relevantes en el contexto actual, como los estudios elaborados por los sindicatos docentes y los organismos internacionales. Pese a lo anterior, el trabajo contribuye al debate en torno a la salud/enfermedad docente a partir de los hallazgos de la investigación internacional que pueden ser utilizados en el diseño de acciones pertinentes a la realidad local; se reconstruyen los estudios sobre salud y condiciones de trabajo docente en el escenario actual, permitiendo articular los conocimientos académicos producidos en el último año, a fin de distinguir corrientes analíticas que ofrezcan lecturas renovadas acerca de la docencia en tiempos de emergencia sanitaria. Si bien, en el artículo se alude a las enfermedades tradicionalmente vinculadas a la docencia, el énfasis se centra en develar las implicaciones de la pandemia en la salud docente como tópicos emergentes en el campo académico internacional, como la irrupción de molestias físicas derivadas del uso prologado de la computadora, padecimientos emocionales resultado del confinamiento y la incertidumbre, así como aflicciones de tipo laboral vinculadas con la sobrecarga de trabajo y la constante fiscalización respecto del quehacer docente.

Del análisis se concluye que los estudios sobre la docencia en tiempos de pandemia se centran en las implicaciones de la COVID-19 en la salud y vida cotidiana del profesorado, así como en las estrategias de intervención para su bienestar físico y mental. Sin embargo, dado que la salud/enfermedad es un fenómeno complejo y de múltiples aristas (Scribano, 2012), es necesario ampliar las investigaciones con una mirada pluriparadigmática y multidisciplinar que indague respecto de la experiencia del profesorado en contextos de vulnerabilidad, su participación en iniciativas de autocuidado, su incidencia según la información de los sistemas de salud, el papel de los sindicatos, además, siguiendo la propuesta de Trinidad (2021), el análisis de las decisiones de docentes y directores en momentos de crisis. Considerando los acontecimientos recientes, se prevé la discusión en torno a los planes de vacunación, la respuesta del profesorado ante la inoculación y su papel en la promoción de la vacuna entre las comunidades.

A finales del 2020, organismos internacionales como el Fondo de las Naciones Unidas para la Infancia (UNICEF), la Internacional de la 
Educación (IE) y la UNESCO exhortaban a los gobiernos a dar acceso prioritario a los docentes en la vacunación contra la COVID-19 (IE, 2020; UNICEF, 2020). Hoy, los avances en el rubro son desiguales: en Italia, el profesorado formó parte de la primera ola de vacunación -a la par del personal médico y el grupo de adultos mayores-; en Alemania, Argentina, Chile, España y Estados Unidos la inoculación comenzó a mediados de febrero, luego de atender al personal sanitario de primera fila y a las personas mayores; en Portugal, los docentes fueron incluidos entre los grupos prioritarios, sin embargo, la vacuna aún no se ha iniciado; en Perú, incluir al profesorado en la primera fase de vacunación constituye un debate abierto; mientras, en Bolivia y Francia hasta el momento los docentes aparecen al margen de los planes de vacunación. Por su parte, en Finlandia, Noruega y Suecia la vacunación del profesorado ocupa un lugar secundario en la lucha contra la pandemia (Infobae, 2021). En México, la vacunación del personal docente se declaró prioridad sanitaria ante la apertura de los Centros Comunitarios de Aprendizaje (CCA) y el regreso a las actividades escolares presenciales; como parte del reconocimiento social "al trabajo, compromiso, esfuerzo, sacrificio y dedicación [de los maestros] durante la pandemia" (Secretaría de Educación Pública [SEP], 2021, párr. 12). Sin embargo, su aplicación está determinada por las condiciones epidemiológicas de cada entidad.

El acceso a la vacuna pone de manifiesto la desigualdad entre los países, la que se materializa en la capacidad de gestión de los gobiernos, su poder adquisitivo y capital científico-tecnológico ante la escasez de vacunas, situación clave en los planes de reapertura escolar. La cartografía de vacunación contra la COVID-19 ofrece un panorama inicial de las políticas docentes dirigidas al cuidado de la salud. Mientras en los países con mayor riqueza (Alemania, Canadá, Estados Unidos, Francia, Italia, Japón y Reino Unido, que solo albergan al $10 \%$ de la población), la vacunación es una realidad; en las naciones con menos recursos se apela a la distribución equitativa de las vacunas en medio de actos de corrupción para alterar los padrones de inoculación y el suministro de vacunas de aire (AFP, AP y Reuters, 2021). Con todo, la vacuna constituye una solución parcial contra el virus que, requiere ser acompañada de políticas de salud integrales, permanentes y equitativas. 


\section{Referencias}

Abanades, M. (2020). La crisis de salud, de bienestar emocional y de competencias para ser un buen docente. Revista de Coтunicación y Salud, 10(2), 249-263. https://doi.org/10.35669/ rcys.2020.10(2).249-263

AFP, AP y Reuters. (21 de febrero de 2021). Acaparan 7 países la mitad de vacunas COVID disponibles. La Jornada. https://www. jornada.com.mx/notas/2021/02/21/mundo/acaparan-7-paises-la-mitad-de-vacunas-covid-disponibles/

Aperribai, L., Cortabarria, L., Aguirre, T., Verche, E., y Borges, Á. (2020). Teacher's physical activity and mental health during lockdown due to the COVID-2019 pandemic. Frontiers in Psychology, (11), 1-14. https://doi.org/10.3389/fpsyg.2020.577886

Bravo, N., Mansilla, J. G., y Véliz, A. (2020). Teletrabajo y agobio laboral del profesorado en tiempos de COVID-19. Medisur, 18(5), 998-1008. http://www.medisur.sld.cu/index.php/medisur/ article/view/4732

Burbano, V. M., Valdivieso, M. A., y Burbano, Á. S. (2020). Teletrabajo académico afectado por el coronavirus. Una mirada desde un grupo focal de profesores universitarios. Espacios, 41(42), 335-348. https://doi.org/10.48082/espacios-a20v41n42p29

Casimiro, W. H., Casimiro, C. N., Barbachán, E. A., y Casimiro, J. F. (2020). Stress, anguish, anxiety and resilience of university teachers in the face of COVID-19. Utopía y Praxis Latinoamericana, 25(1), 453-464. https://doi.org/10.5281/zenodo.4009790

Çifçi, F. y Demir, A. (2020). The effect of home-based exercise on anxiety and mental well-being levels of teachers and pre-service teachers in COVID-19 pandemic. African Educational Research Journal, 8(Special), 20-28. https://doi.org/10.30918/ AERJ.8S2.20.029

Codina, L. (2020). Cómo hacer revisiones bibliográficas tradicionales o sistemáticas utilizando bases de datos académicas. Revista ORL, 11(2), 139-153. http://dx.doi.org/10.14201/orl.22977

Comisión Nacional para la Mejora Continua de la Educación, MEJOREDU. (2020). Indicadores nacionales para la mejora continua de la educación en México 2020. Cifras del ciclo escolar 2018-2019. MEJOREDU. https://www.mejoredu.gob.mx/images/publicaciones/indicadores-nacionales.pdf 
Cornejo, R., Araya, R., Parra, D., y Vargas, S. (18 de agosto de 2020). El bienestar/malestar emocional de los/as docentes y la "bitácora" del abandono. Diario Uchile [En línea]. https://radio. uchile.cl/2020/08/18/el-bienestarmalestar-emocional-delosas-docentes-y-la-bitacora-del-abandono/

Cruze, W. W. (1949). Mental hygiene and in-service-training for teachers. Education, 69(5), 288-292.

Day, C. (2019). Educadores comprometidos. Qué son, qué hacen, por qué lo hacen y lo que verdaderamente importa. Narcea.

Dayal, H. C. y Tiko, L. (2020). When are we going to have the real school? A case study of early childhood education and care teachers' experiences surrounding education during the COVID-19 pandemic. Australasian Journal of Early Childhood, 45(4), 336-347. https://doi.org/10.1177/1836939120966085

Fondo de las Naciones Unidas para la Infancia, UNICEF. (15 de diciembre de 2020). Los maestros deben tener prioridad en la vacunación contra la COVID-19. UNICEF. https://www.unicef. org/es/comunicados-prensa/maestros-deben-tener-prioridad-vacunacion-contra-covid19

Gañán, A., Correa, J. J., Ochoa, S. A., y Orejuela, J. J. (2020). Tecnoestrés laboral derivado de la virtualidad obligatoria por prevención del COVID-19 en docentes universitarios de Medellín (Colombia). Trabalho (En)Cena, 5(2), 1-23. https://doi. org $/ 10.20873 / 2526$

Grant, M. J. y Booth, A. (2009). A typology of reviews: an analysis of 14 review types and associated methodologies. Health Information and Libraries Journal, 26(2), 91-108. https://doi. org/10.1111/j.1471-1842.2009.00848.x

Inciarte, A., Paredes-Chacín, A. J., y Zambrano, L. M. (2020). Docencia y tecnologías en tiempos de pandemia COVID-19. Utopía y Praxis Latinoamericana, 25(Extra 8), 195-215. http://doi. org/10.5281/zenodo.4087411

Infobae. (13 de marzo de 2021). Así avanza la vacunación a los docentes contra el coronavirus en el mundo. Infobae. https://www. infobae.com/america/mundo/2021/03/13/asi-avanza-lavacunacion-contra-el-coronavirus-a-los-docentes-en-elmundo/

Internacional de la Educación, IE. (14 de diciembre de 2020). La Internacional de la Educación y la Unesco piden que se dé acceso 
prioridad a los docentes y las docentes en la vacunación contra la COVID. IE. https://www.ei-ie.org/spa/detail/17079/ la-internacional-de-la-educaci\%C3\%B3n-y-la-unesco-piden-que-se-d\%C3\%A9-acceso-prioridad-a-los-docentes-ylas-docentes-en-la-vacunaci\%C3\%B3n-contra-la-covid

Karakaya, F., Adıgüzel, M., Üçüncü, G., Çimen, O., y Yilmaz, M. (2021). Teachers' views towards the effects of COVID-19 pandemic in the education process in Turkey. Participatory Educational Research, 8(2), 17-30. http://dx.doi.org/10.17275/ per.21.27.8.2

Kayabınar, E., Kayabınar, B., Önal, B., Zengin, H. Y., y Köse, N. (2020). The musculoskeletal problems and psychosocial status of teachers giving online education during the COVID-19 pandemic and preventive telerehabilitation for musculoskeletal problems. Work, 68(1), 33-43. https://doi.org/10.3233/ WOR-203357

Kong, X., Zheng, K., Tang, M., Kong, F., Zhou, J., Diao, L., Wu, S., Jiao, P., Su, T., y Dong, Y. (2020). Prevalence and factors associated with depression and anxiety of hospitalized patients with COVID-19. medRxiv. The Preprinter Server for Health Sciences. https://doi.org/10.1101/2020.03.24.20043075

Matiz, A., Fabbro, F., Paschetto, A., Cantone, D., Paolone, A. R., y Crescentini, C. (2020). Positive impact of mindfulness meditation on mental health of female teachers during the COVID-19 outbreak in Italy. International Journal of Environmental Research and Public Health, 17(18), 1-22. https://doi. org/10.3390/ijerph17186450

Meo, A. I. y Dabenigno, V. (2021). Teletrabajo docente durante el confinamiento por COVID-19 en Argentina. Condiciones materiales y perspectivas sobre la carga de trabajo, la responsabilidad social y la toma de decisiones. RASE. Revista de Sociología de la Educación, 14(1), 103-127. https://doi. org/10.7203/RASE.14.1.18221

Miles, M. B., Huberman, A. M., y Saldaña, J. (2014). Qualitative data analysis. A methods sourcebook. SAGE Publications.

Organización de las Naciones Unidas para la Educación, la Ciencia y la Cultura, UNESCO. (27 de marzo de 2020). Respuesta frente al brote del COVID 19. Llamado a la acción respecto a los y las docentes. UNESCO. https://teachertaskforce.org/sites/ 
default/files/migrate_default_content_files/ttf_covid19_ call\%20to\%20action_27mar2020_esp_1.pdf

Organización Mundial de la Salud, OMS. (2019). Constitución. OMS. https://www.who.int/es/about/who-we-are/constitution

Organización para la Cooperación y el Desarrollo Económicos, OCDE. (2018). Políticas docentes efectivas. Conclusiones del informe PISA. OCDE. http://dx.doi.org/10.1787/9789264301603-en

Ozamiz-Etxebarria, N., Berasategi, N., Idoiaga, N., y Dosil, M. (2021a). The psychological state of teachers during the COVID-19 crisis: The challenge of returning to face-to-face teaching. Frontiers in Psychology. https://doi.org/10.3389/fpsyg.2020.620718

Ozamiz-Etxebarria, N., Dosil, M., Idoiaga, N., y Berasategi, N. (2021b). Estado emocional del profesorado de colegios y universidades en el norte de España ante la COVID-19. Revista Española de Salud Pública, (95), 1-8. https://www.mscbs.gob.es/ biblioPublic/publicaciones/recursos_propios/resp/revista_ cdrom/VOL95/O_BREVES/RS95C_202102030.pdf

Parra, M. (2005). Análisis comparativo. En M. Robalino y A. Körner (Eds.), Condiciones de trabajo y salud docente. Estudio de casos en Argentina, Chile, Ecuador, México, Perú y Uruguay (pp. 21-42). OREALC/UNESCO. https://unesdoc.unesco. org/ark:/48223/pf0000142551_spa

Pérez, C., Contreras, J., y Mattoli, M. (2020). La conversión a un mundo digital hiperconectado en salud impulsado por la pandemia del COVID-19. Revista médica de Chile, 148(8), 1223-1224. http://dx.doi.org/10.4067/S0034-98872020000801223

Pinheiro Almeida, L., Moreira Cruz, E., Brito Alexandre, T., Vasconcelos Carneiro, S. N., Vasconcelos Carneiro, S., Oliveira Bezerra, M., Nunes Maia, A., y Farias Câmara, C. (2021). Saúde mental docente: um olhar para o profissional da rede pública de ensino. Brazilian Journal of Development, 7(2), 14769-14786. https://doi.org/10.34117/bjdv7n2-211

Prada, R., Gamboa, A. A., y Hernández, C. A. (2021). Efectos depresivos del aislamiento preventivo obligatorio asociados a la pandemia del COVID-19 en docentes y estudiantes de una universidad pública en Colombia. Psicogente, 24(45), 1-20. https://doi.org/10.17081/psico.24.45.4156

Ramos-Huenteo, V., García-Vásquez, H., Olea-González, C., LobosPeña, K., y Sáez, D. (2020). Percepción docente respecto al 
trabajo pedagógico durante la COVID-19. Ciencia América, 9(2), 1-20. http://dx.doi.org/10.33210/ca.v9i2.325

Robalino, M. (2012). La salud y el trabajo en la educación de Latinoamérica. Revista Retratos de la Escuela, 6(11), 315-326. http:// retratosdaescola.emnuvens.com.br/rde/article/down$\operatorname{load} / 234 / 418$

Robalino, M. y Körner, A. (2005). Condiciones de trabajo y salud docente. Estudio de casos en Argentina, Chile, Ecuador, México, Perú y Uruguay. OREALC/UNESCO. https://unesdoc.unesco.org/ark:/48223/pf0000142551_spa

Robinet-Serrano, A. L. y Pérez-Azahuanche, M. (2020). Estrés en los docentes en tiempos de pandemia COVID-19. Polo del conocimiento, 5(12), 637-653. https://polodelconocimiento.com/ ojs/index.php/es/article/view/2111/4197

Rojas, O., Martínez, M., y Riffo, R. (2020). Gestão diretiva e estresse laboral do profissional docente: um olhar a partir da pandemia COVID-19. Revista on line de política e gestão educacional, 24(3), 1226-1241. https://doi.org/10.22633/rpge.v24i3.14360

Roman, T. A. (2020). Supporting the mental health of teachers in COVID-19 through trauma-informed educational practices and adaptive formative assessment tools. Journal of Technology and Teacher Education, 28(2), 473-481. https://www.researchgate.net/publication/348923263

Sahu, P. (2020). Closure of universities due to coronavirus disease 2019 (COVID-19): Impact on education and mental health of students and academic staff. Cureus, 12(4), 1-5. https://doi. org/10.7759/cureus.7541

Sánchez, M., Martínez, A. M., Torres, R., de Agüero, M., Hernández, A. K., Rendón, V. J., y Jaimes, C. A. (2020). Retos educativos durante la pandemia de COVID-19: una encuesta a profesores de la UNAM. Revista Digital Universitaria, 21(3), 1-24. http://doi.org/10.22201/codeic.16076079e.2020.v21n3.a12

Scribano, A. (2012). Sociología de los cuerpos/emociones. Revista Latinoamericana de Estudios sobre Cuerpos, Emociones y Sociedad, (10), 93-113. http://www.relaces.com.ar/index.php/ relaces/article/view/237/234

Secretaría de Educación Pública, SEP. (22 de enero de 2021). Boletín $N^{o} 17$ Inicia este fin de semana el proceso de vacunación para personal docente y administrativo en el estado de Campeche: 
SEP. Gobierno de México. https://www.gob.mx/sep/es/articulos/boletin-no-17-inicia-este-fin-de-semana-el-procesode-vacunacion-para-personal-docente-y-administrativoen-el-estado-de-campeche-sep?idiom=es

Sibaja, Z., Hernández, N., y Granados, R. Á. (2020). Reinventando la práctica docente en la Universidad Estatal a Distancia de Costa Rica: una oportunidad de crecimiento profesional en tiempos de COVID-19. Innovaciones Educativa, 22(Especial), 129-142. https://doi.org/10.22458/ie.v22iEspecial.3245

Sieglin, V. y Ramos, M. E. (2007). Estrés laboral y depresión entre maestros del área metropolitana de Monterrey. Revista Mexicana de Sociologia, 69(3), 517-551. https://www.jstor.org/stable/20454302

Stachteas, P. y Stachteas, C. (2020). The psychological impact of the COVID-19 pandemic on secondary school teachers. Psychiatriki, 31(4), 293-301. https://doi.org/10.22365/jpsych.2020.314.293

Trinidad, J. E. (2021). Equity, engagement, and health: school organizational issues and priorities during COVID-19. Journal of Educational Administration y History, 53(1), 67-80. https:// doi.org/10.1080/00220620.2020.1858764

Zamora-Díaz, W.-J., Cobos-Sanchiz, D., y López-Noguero, F. (2017). Condiciones sociales y salud laboral del profesorado nicaragüense de secundaria. Revista de Pedagogía, 38(103), 192-208. http:// saber.ucv.ve/ojs/index.php/rev_ped/article/view/14933 